\title{
Local/global non-intrusive crack propagation simulation using a multigrid $\mathrm{X}$-FEM solver
}

\author{
Jean-Charles Passieux · Julien Réthoré · Anthony Gravouil · Marie-Christine \\ Baietto
}

Received: date / Accepted: date

\begin{abstract}
A Local/Global non-intrusive coupling algorithm is proposed for the analysis of mixed-mode crack propagation. It is based on a three scale multigrid and extended finite element method, that was proposed recently for the direct estimation of stress intensity factors of static cracks. The algorithm couples a linear elastic global model (possibly performed by a industrial software) with an enhanced local model capable of modeling a crack and accurately estimating SIFs (performed by a separate research code). It is said non-intrusive since it does not modify the global mesh, its connectivity and solver. For the global model, the contribution of the local patch consists in additional nodal efforts near the crack, which makes it compatible with most softwares. Further the shape of the domain over which the local model is applied is automatically adapted during propagation.
\end{abstract}

Keywords nested models $\cdot$ mixed mode $\cdot$ localized multigrid $\cdot$ williams $\cdot$ stress intensity factors

\section{Introduction}

The question of the inclusion of a crack and its propagation in a finite element model initially not expected for this, is a question which is still today the subject of numerous

\section{J.-C. Passieux}

Université de Toulouse, Institut Clément Ader (ICA)

INSA de Toulouse, UPS, Mines Albi, ISAE.

135 av de Rangueil, 31077 Toulouse Cedex, France

E-mail: passieux@insa-toulouse.fr

J. Réthoré, A. Gravouil, M.-C. Baietto

Laboratoire de Mécanique de Contacts et des Structures (LaMCoS), INSA de Lyon/CNRS UMR5259/Université de Lyon.

20, avenue des Sciences, F-69621 Villeurbanne Cedex, France

E-mail: julien.rethore, anthony.gravouil, marie-christine.baietto@insalyon.fr studies. The latter does not only aim at accurately simulating the presence and propagation of cracks. Indeed, a special effort is dedicated to the development of tools increasingly generic, flexible and simple to implement and to use in a constrained environment.

The extended finite element method (X-FEM [27]) was originally developed to overcome the high intrusiveness of remeshing based methods [10] that may have meshing constraints near the the crack front singularity [2]. X-FEM can account for both the displacement discontinuity across the crack faces and the near-tip crack singularities, by adding analytical enrichment functions to the classical finite element approximation subspace. It is then possible to model a crack with a mesh which does not conform to it. For crack growth simulation, remeshing is also no longer required at each propagation step. In this sense, X-FEM has achieved a first step towards clearly less intrusive simulation of fracture problems.

To go a step forward, an alternative method (HAX-FEM) was proposed recently [37,29]. It is based on X-FEM far from the crack tip. Only the singular enrichment near the crack tip differs. The idea consists in unactivating the standard finite element interpolation around the crack tip and replace it by analytical modes taken from the linear elastic crack tip asymptotic fields [40]. The latter provide directly reliable estimates of the stress intensity factors (SIF) and higher order terms. The coupling between X-FEM and analytical models is performed by an overlapping Arlequin coupling [37] or a Mortar like integral matching on the interface [29]. This method is able to accurately estimate SIF without resorting to a posteriori recorvery techniques like least-squares, energy integrals (i.e. interaction integrals) or configurational forces [31].

A problem of industrial practical importance and of large impact is that the initial CAD mesh is such that there is more than one order of magnitude between the characteristic di- 
mensions of the structure and the crack. It this case, even with the introduction of singular and discontinuous enrichments, the initial mesh may not be fine enough to simulate accurately the presence of the crack [35]. For that purpose, many multiscale techniques have been developed to couple the scale of the structure (and its initial mesh) to the scale of the crack. For instance, coupling algorithms are based on domain decomposition [19,26,4], on multigrid algorithms $[35,15]$, on extended/generalized finite elements [24,32], on multiscale projection [21], on patch methods through Arlequin coupling [37,3] or on integral matching [9,29]. The latter has the advantage to avoid blending elements. Similarly, HAX-FEM method was associated with a localized multigrid algorithm in [29].

However, these innovative methods are generally very intrusive, which represents an obstacle to their deployment to large scale industrial applications.

To reduce this high intrusiveness, a new family of nonintrusive coupling algorithms has recently been pioneered by Gendre et al. [12]. Theses methods are dedicated for global (possibly large scale) finite element model, integrated within a commercial software, in which a local phenomenon exists, but that the global model is not able to account for. It may be just a local loading requiring local mesh refinement $[16,25,26]$, but also the occurrence of a local nonlinearity (plasticity [12]). The idea is to develop a local/global coupling algorithm while avoiding any modification of the industrial code used to handle the global problem. Neither the mesh nor the solver are modified. This constraint, though restrictive, nevertheless allows one to couple industrial sofware with research demonstrator code [1].

These iterative methods are based on algorithms originally developed for domain decomposition (DD) $[12,13]$. The purpose of this paper is to propose, respecting the constraint of non-intrusiveness of the global problem, an alternative algorithm based on a localised multigrid algorithm for the simulation of mixed-mode crack propagation. It is shown how to use both X-FEM or DEK-FEM in a globally linear elastic finite element model processed by a code considered as a black box. Curvilinear crack propagation is also considered. The shape of the domain over which the local model is applied is automatically updated as well as the additional enrichments whereas the global model remains the same, with identical connectivity, operator and solver during propagation.

The paper is organized as follows. Section 1 presents the proposed multigrid-based coupling algorithm in comparison with the DD-based non-intrusive solver of [12]. In this section only FEM/FEM coupling is considered. Section 2 is devoted to the extension of the method to achieve global FEM/local X-FEM non-intrusive coupling, with different intergrid operators. It is also shown that the stress intensity factor estimation can be obtained directly by the addition of an optional third analytical grid. A static straight crack synthetic example (sec.3) and two curvilinear crack propagation realistic simulations (sec. 4 and 5) are proposed to illustrate the accuracy of the method and the interest of nonintrusiveness in the context of the correlation between numerical simulation and experimentation.

\section{Localized multigrid and non-intrusive coupling}

In this section, the localised multigrid algorithm is shown to be a good candidate for local/global non-intrusive coupling. In order to make comparisons, this section is limited to the analysis of the coupling of two linear elastic FEM models.

\subsection{Localized multigrid solver (LMS)}

Multigrid methods [7,28] are based on the fact that for many iterative solvers (Jacobi, Gauss-Seidel, Krylov solvers...), the short-varying part of the solution is captured after very few iterations, whereas it usually takes much more iterations to get the large variations accurately. This is the socalled error smoothing property. To accelerate this second convergence phase, multigrid algorithms propose to transfer the residual on a hierarchy of coarser grids which are more likely to compute the low frequency part of the solution. This process is completed until the mesh is coarse enough for a direct solver to be used. Then, the solution on the finer grids are successively improved with the smooth corrections (prolongation operation), in order to accelerate the convergence of the iterative algorithm on finer grids. This scheme, called the multigrid cycle, is completed once per iteration until convergence. A prolongation operator $\mathbf{P}$ (resp. restriction $\mathbf{R}$ ) is introduced to transfer the displacement (resp. residual) from the coarse mesh to the fine (resp. fine to coarse). Their construction is particularly trivial when using nested meshes [28].

For the sake of simplicity, let us consider a two-level Localized MultiGrid (LMG) algorithm defined on the grids of Figure 1, made of one global coarse mesh $\mathscr{M}_{G}$ and one lo-

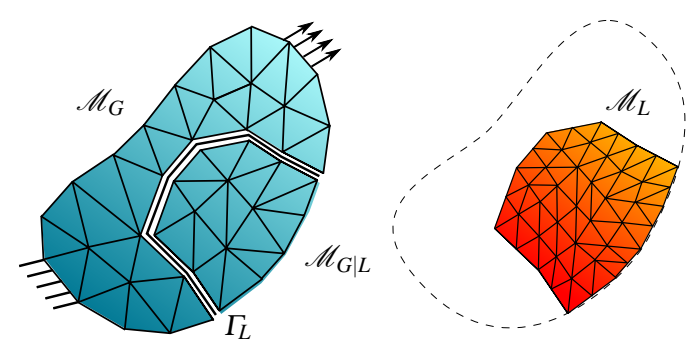

Fig. 1 A 2-grid hierarchy: Global coarse mesh $\mathscr{M}_{G}$ and local fine mesh $\mathscr{M}_{L}$

calized fine mesh $\mathscr{M}_{L}$. The region overlapped by the local 
model in the global mesh is denoted $\mathscr{M}_{G \mid L}$. The intersection between its boundary and $\mathscr{M}_{G}$ reduces to an interface $\Gamma_{L}$. The quantity $\square_{G}$ (resp. $\square_{L}$ ) is the discretization of $\square$ on Mesh $\mathscr{M}_{G}$ (resp. $\mathscr{M}_{L}$ ). The quantity $\square_{G \mid L}$ defines the discretization of $\square$ on $\mathscr{M}_{G \mid L}$ and padded with zeros, such that $\square_{G}$ and $\square_{G \mid L}$ have the same size.

One multigrid iteration is made of three sub-stages:

1. The global coarse problem which reads:

$$
\mathbf{K}_{G} \mathbf{U}_{G}^{k+1}=\mathbf{F}_{G}-\mathbf{P}^{T} \mathbf{K}_{L} \mathbf{U}_{L}^{k}+\mathbf{K}_{G \mid L} \mathbf{U}_{G}^{k}
$$

where $\mathbf{K}$ stands for the stiffness matrix, $\mathbf{U}$ the dof vector and $\mathbf{F}$ the generalized force vector. The right-hand-side of this problem involves the loading $\mathbf{F}_{G}$ and two additional terms whose role is to inactivate the coarse stiffness $\mathbf{K}_{G \mid L} \mathbf{U}_{G}^{k}$ and replace it by the restriction of the fine $\mathbf{K}_{L} \mathbf{U}_{L}^{k}$. This global problem is solved exactly.

2. The local solution $\mathbf{U}_{L}^{k}$ is improved by the prolongation of the coarse correction:

$$
\mathbf{U}_{L}^{k}=\mathbf{U}_{L}^{k}+\mathbf{P}\left(\mathbf{U}_{G}^{k+1}-\mathbf{U}_{G}^{k}\right)
$$

3. This corrected solution is then smoothed by few iterations (usually less than five) of the following fine local problem:

$$
\mathbf{K}_{L} \mathbf{U}_{L}^{k+1}=\mathbf{F}_{L} \quad \text { with }\left.\mathbf{U}_{L}^{k+1}\right|_{\Gamma_{L}}=\left.\mathbf{P} \mathbf{U}_{G}^{k+1}\right|_{\Gamma_{L}}
$$

with the coarse displacement $\mathbf{U}_{G}$ prescribed on its boundary $\Gamma_{L}$.

These 3 steps are performed until convergence. For more details, the reader is referred to $[28,35]$. This algorithm yields the solution of the global coarse model in which its local part $\mathscr{M}_{G \mid L}$ is replaced by the fine local model $\mathscr{M}_{L}$.

\subsection{Comparison with non-intrusive coupling}

Let us imagine that the coarse grid stands for a global model and the finer grid for a local model. Thus, one can notice that this LMS can be used for non-intrusive coupling since the different global solutions (1) are performed with the same operator $\mathbf{K}_{G}$. Indeed, the global problem only involves a special right hand side, which can be seen, for a commercial code, as an additional nodal loading localized on $\mathscr{M}_{G \mid L}$ as performed in [12]. The two sub-stages (2) and (3) can be achieved using another code. Note that at this stage, the localized multigrid algorithm can be seen as a non-intrusive refinement method.

In order to compare the algorithms proposed by [12] and the LMS one, let us consider a case for which both local and global models are assumed linear elastic, with the same interpolations (so that we can no longer speak of fine and coarse meshes, but of local and global ones). The only difference concerning the LMS algorithm is that the prolongation and restriction operators are identity matrices. In this case, both algorithms are very similar, but some differences remain:

1. The supplementary loading added to the right hand side of the global problem is defined at the degrees of freedom located at the interface only in [12], whereas it may be defined over the whole overlapped region using the localized multigrid algorithm.

2. The local problem is solved exactly in [12] (at least in its basic version), whereas only few smoothing iterations are performed in the case of a multigrid solver.

Both differences come from the nature of the underlying algorithm, domain decomposition in [12] and multigrid in the second.

Finally, the LMS can be seen as an alternative to the work of Gendre et al. [12] for non-intrusive coupling. It has the advantage to be ready for nested models, as multigrid solvers generally involve more than two grids. In the following section we propose to investigate the use of the localized multigrid solver DEK-FEM [29] to perform non-intrusive simulation of mixed-mode crack propagation.

\section{Non-intrusive coupling based on X-FEM}

Up to now, the models used on both domains are based on classical FEM.

In this section, the LMS is used for the non-intrusive coupling between a FEM model holding over $\mathscr{M}_{G}$ global domain with a X-FEM model holding over $\mathscr{M}_{L}$ local domain. This aims at solving large-scale problems by splitting the scales with appropriate models and refinements.

Let us consider a problem with a small propagating crack. A FEM-based model is employed over the global domain, which plays the role of the coarser grid (see Fig. 2). The coarse grid involves only standard finite elements. An

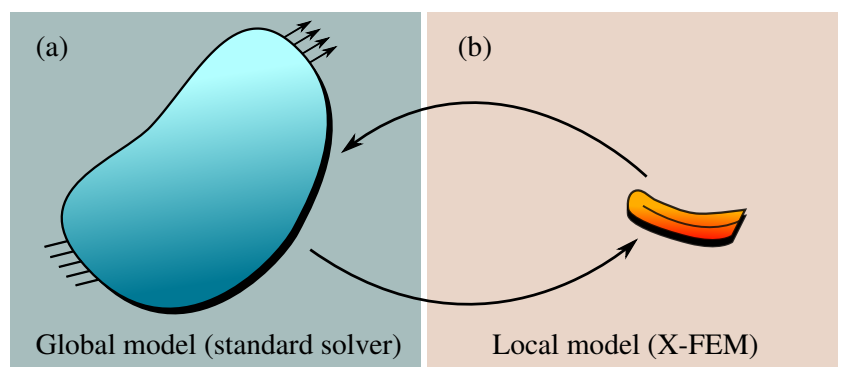

Fig. 2 X-FEM Multigrid-based non-intrusive coupling technique. The iterative scheme involves global resolution with a standard solver (blue) and few smoothing iterations on the local X-FEM model (orange) 
$\mathrm{X}$-FEM based model is then introduced over the localized domain, the finer grid, to describe the crack. It is obviously located around the crack and its shape depends on the crack geometry as we will see in Section 3.3. Like any other localized multigrid algorithm, the finer grids replace the coarser ones in the model such that, after convergence, the multiscale solution at a point $x$ is defined by the displacement fields associated with the finest grid existing at that point $x$. Like [12], it is more a model substitution rather than a local model enrichment [16].

Remark. In [35], it is shown that if one wants to minimize the number of multigrid iterations, the grids whose elements are larger than the size of the crack should use standard FEM only. The choice made here to enrich the local grid, but not the global one, is driven by the nonintrusiveness condition and may therefore not be optimal in that sense.

The prolongation and restriction operators are described in section 3.1 .

\subsection{Restriction and prolongation operators}

The multigrid iterations used to perform the X-FEM multigrid algorithm are the same as the ones presented in section 2. An iteration is divided into three sub-stages

1. (1) a global standard FE based problem,

2. (2) one update of the local solution,

3. (3) and one local X-FEM based problem.

The only difference introduced by the X-FEM use lies in the expression of the restriction and prolongation operators. The collocation method presented in details in $[36,35]$ is here followed. Some of the main aspects are recalled in this section.

The prolongation operator transfers nodal displacements from the coarse mesh $\mathscr{M}_{G}$ to the fine grid $\mathscr{M}_{L}$. If the latter are nested and if standard FE are used on both meshes, then an exact transfer is obtained thanks to:

$u_{n}^{L}=\sum u_{i}^{G} N_{i}^{G}\left(x_{n}\right)$

where $x_{n}^{L}$ is the position of the node associated with the degree of freedom $u_{n}^{L}$ in the fine (or local) mesh. $N_{i}^{G}$ denotes the coarse (or global) shape function and $u_{i}^{G}$ the associated dof.

According to X-FEM, the displacement interpolation reads [27]:

$$
\begin{aligned}
u^{L}(x)=\sum_{i \in N} u_{i}^{L} N_{i}^{L}(x)+\sum_{i \in N_{h}} a_{i}^{L} N_{i}^{L}(x) H(x) & \\
& +\sum_{i \in N_{s}} \sum_{j=1}^{4} b_{i j}^{L} N_{i}^{L}(x) F_{j}(x)
\end{aligned}
$$

where $N, N_{h}$ and $N_{s}$ define the whole set of nodes, the set of nodes with heaviside enrichment $H$ and the set of nodes with singular enrichment functions $F_{j}$, respectively. $u_{i}^{L}, a_{i}^{L}$ and $b_{i j}^{L}$ denote the regular degrees of freedom and those associated with heaviside and singular enrichments functions, respectively.

Since $\mathscr{M}^{G}$ is based on FEM and $\mathscr{M}_{L}$ on X-FEM, it is not always possible to define an exact collocation transfer everywhere. In this case, relation (4) becomes:

$u_{n}^{L}+a_{i}^{L} H\left(x_{n}\right)+\sum_{j=1}^{4} b_{i j}^{L} F_{j}\left(x_{n}\right)=\sum u_{i}^{G} N_{i}^{G}\left(x_{n}\right)$

In this situation, and more generally, when at least one grid supports enrichments, the empirical method proposed in [36] is followed. It consists in performing a separate transfer of the degrees of freedom. For instance, if a node is enriched on the local model and not in the global one, the corresponding degrees of freedom are set to zero. In [36], it is shown that the transfert errors, as compared to a mortar tranfer operator, are small and localized in small regions close to enrichments. The error introduced has then a high frequency content which is corrected after few smoothing iterations. This approximate collocation method is used is the sequel as it is more convenient and very less time consuming than the Mortar method and it provides substantially the same results [35].

In this section, we considered the coupling of a global FEM model with a local model based on standard X-FEM. In fact, any enhanced X-FEM models (for instance with contact of the lips [33]) can be coupled to a global FEM model with the very same technique. In the next section, it is shown that an X-FEM variant [30], that include direct SIF estimation, can be used by simply adding a special grid around the tip.

\subsection{Direct estimation of SIFs}

Most propagation laws are based on stress intensity factors (SIFs). Unfortunately, classical X-FEM is not able to provide directly reliable estimates of SIFs. The latter usually require post-processing techniques (least square, energy related techniques... ). In [37] an alternative tip enrichment technique was proposed to provide directly accurate estimates of the stress intensity factors and higher order terms. In this section, this technique is used in a non-intrusive way, based on the algorithm of [29], by the addition of a third grid localized at the crack tip.

The method consists in desactivating the regular shape functions and replacing them by an analytical reduced basis taken from the linear elastic fracture mechanics, known as the Williams' expansion. 
Let us consider a linear elastic isotropic 2D domain with a semi-infinite straigh crack where $r$ and $\theta$ define the local co-ordinates at the crack tip. Then the displacement field $\mathbf{u}(r, \theta)$ can be written as the following double series [40]:

$\mathbf{u}(r, \theta)=\sum_{i=I, I I} \sum_{n=0}^{\infty} a_{i}^{n} \Phi_{i}^{n}(r, \theta)$

where $a_{i}^{n}$ are the coefficients associated with the crack tip asymptotic fields $\Phi_{i}^{n}$ whose expression reads:

$\Phi_{I}^{n}(r, \theta)=r^{n / 2} \mid \begin{aligned} & \left(\kappa+\frac{n}{2}+(-1)^{n}\right) \cos \frac{n \theta}{2}-\frac{n}{2} \cos \frac{(n-4) \theta}{2} \\ & \left(\kappa-\frac{n}{2}-(-1)^{n}\right) \sin \frac{n \theta}{2}+\frac{n}{2} \sin \frac{(n-4) \theta}{2}\end{aligned}$

$\Phi_{I I}^{n}(r, \theta)=r^{n / 2} \mid \begin{aligned} & \left(-\kappa-\frac{n}{2}+(-1)^{n}\right) \sin \frac{n \theta}{2}+\frac{n}{2} \sin \frac{(n-4) \theta}{2} \\ & \left(\kappa-\frac{n}{2}+(-1)^{n}\right) \cos \frac{n \theta}{2}+\frac{n}{2} \cos \frac{(n-4) \theta}{2}\end{aligned}$

in which the Kolossov constant $\kappa$ is equal to $\kappa=3-4 v$ in plane strain and $\kappa=((3-v) /(1+v))$ in plane stress condition. $v$ denotes the Poisson's ratio.

These asymptotic modes have some remarkable properties. On the one hand, coefficients $a_{I}^{0}, a_{I I}^{0}$ and $a_{I I}^{2}$ are the rigid body modes and correspond respectively to the translation along the crack direction, translation in the orthogonal direction and the rotation with respect to the crack tip. On the other hand, $a_{I}^{2}$ is linked to the $T$-stress component in mode $I$. Last, the first order coefficients $(n=1) a_{I}^{1}$ and $a_{I I}^{1}$ are proportional to the stress intensity factors (SIF), such that:

$a_{I}^{1}=\frac{K_{I}}{2 \mu \sqrt{2 \pi}} \quad$ and $\quad a_{I I}^{1}=\frac{K_{I I}}{2 \mu \sqrt{2 \pi}}$

where $\mu$ is the second Lamé's coefficient.

This interpolation is used on a little patch corresponding to a small set of elements in the near-tip region denotes $\mathscr{M}_{W}$. It is seen as a second local/global relationship between the local model $\mathscr{M}_{L}$ and the patch $\mathscr{M}_{W}$. Thus, the notations $\square_{W}, \square_{L \mid W}$ of a quantity $\square$ and $\Gamma_{W}$ are defined analogously. As mentionned before, the idea is to remove standard finite elements in this zone and replace them by a Williams' expansion. More precisely, the infinite sum is obviously not required, the expansion is truncated to $m$ terms. In this paper, orders $0 \leq n \leq m=7$ are used. The effect of this truncation on the accuracy of SIF extraction has been adressed in [37, 29]. The remaining X-FEM model and the patch are coupled thanks to Arlequin method [3] over an overlapping area [37] or integral matching on the interface [29]. In [29], it was also shown that it is possible to couple, in a non-intrusive way, this patch to a classical X-FEM model without modifying the X-FEM model. The coupling algorithm is based on coupling algorithm similar to $[12,25]$. It is formulated as follows [29]:
After the initialization phase $\mathbf{U}_{L}^{0}=0, \mathbf{U}_{W}^{0}=0, \Lambda^{0}=$ $0, k=0$, one iteration of the Algorithm 2 is made of the two following steps:

1. X-FEM model on the whole local domain $\mathscr{M}_{L}$ :

$$
\mathbf{K}_{L} \mathbf{U}_{L}^{k+1}=\mathbf{F}_{L}-\mathbf{C}_{L}^{T} \boldsymbol{\Lambda}^{k}+\mathbf{K}_{L \mid W} \mathbf{U}_{L}^{k}
$$

2. Analytical reduced order model on the patch $\mathscr{M}_{W}$ :

$$
\left[\begin{array}{cc}
\mathbf{K}_{W} & -\mathbf{C}_{W}^{T} \\
-\mathbf{C}_{W} & 0
\end{array}\right]\left[\begin{array}{c}
\mathbf{U}_{W}^{k+1} \\
\boldsymbol{\Lambda}^{k+1}
\end{array}\right]=\left[\begin{array}{c}
0 \\
\mathbf{C}_{L} \mathbf{U}_{L}^{k+1}
\end{array}\right]
$$

3. convergence indicator: $\eta=\left\|\mathbf{U}_{W}^{k+1}-\mathbf{U}_{W}^{k}\right\| /\left\|\mathbf{U}_{W}^{k+1}\right\|$

where $\mathbf{C}_{L}$ and $\mathbf{C}_{W}$ are Mortar operators and $\boldsymbol{\Lambda}$ is the Lagrange multiplier used to apply the boundary conditions on the patch $\mathscr{M}_{W}$ (interpolated as described in [29]).

Here, the problem of step 2 (on the analytical patch) is solved exactly, since it is only a $2 n_{W} \times 2 n_{W}$ linear system where $n_{W}$ is the size of Williams' basis (usually less than 15). This algorithm was proved to converge to the exact monoscale (or intrusive) solution [29].

In [29], this algorithm was coupled to the localized multigrid-X-FEM solver [35] thanks to a $W$-multigrid cycle. Following this, the multigrid-based non-intrusive coupling technique for crack propagation simulation can be seen as the association of three models, in order to get direct SIF estimation (Fig. 3). This is typically the case of a nested model.

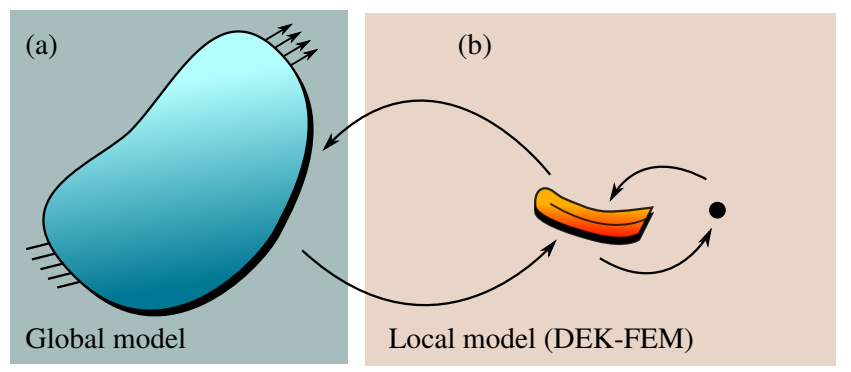

Fig. 3 DEK-FEM-based non-intrusive coupling technique for curvilinear crack growth simulation. The iterative scheme made of global resolution with a standard solver (blue) and few smoothing iterations on the local DEK-FEM model (orange). The local model involves an analytical patch at the crack top (black)

Remark. In this particular case, this association of the two algorithms can be made easier, by noticing that equation (12) can be reformulated as

$\Lambda^{k+1}=-\mathbf{C}_{W}^{-T} \mathbf{K}_{W} \mathbf{C}_{W}^{-1} \mathbf{C}_{L} \mathbf{U}_{L}^{k+1}=-\mathbf{L}_{W} \mathbf{U}_{L}^{k+1}$

were $\mathbf{L}_{W}$ is a kind of Schur complement which yields the reaction force distribution $\Lambda^{k+1}$ of $\mathscr{M}_{W}$ to a prescribed displacement $\mathbf{U}_{L}^{k+1}$ on $\Gamma_{W}$. It is computationally affordable since it corresponds to the inversion of a $n_{W} \times n_{W}$ matrix. 
Next it can be introduced in the coarse problem (11), which reads:

$$
\left(\mathbf{K}_{L}-\mathbf{K}_{L \mid W}+\mathbf{C}_{L}^{T} \mathbf{L}_{W}\right) \mathbf{U}_{L}^{k+1}=\mathbf{F}_{L}
$$

The three-grid $W$-cycle can then be replaced by a two-grid standard $V$-cycle, since both X-FEM and analytical models are solved concurrently by few iterations of the smoothing algorithm on equation (14) within the local model. At the end of the multigrid iterations, one has to compute the displacement in the patch $\mathbf{U}_{W}$ with equation (12). For the user, this technique avoids the choice of the parameter of the $W$ cycle (number of sub-cycles on the local patch per multigrid cycle), while ensuring optimal convergence of the algorithm. According to our numerical tests, the computational time of one multigrid cycle is significantly reduced.

\subsection{Adaptivity of the local model during propagation}

The domain over which the local model is applied, i.e where the grid has to be refined, must surround the crack. In this section we propose to use the level-set representation to adapt the shape of the local model domain versus the crack geometry during propagation.

Two level-sets are used for the implicit description of the crack, following [17]. The first one denoted $\phi$ (called crack level set) is used to represent the discontinuity. The second denoted $\psi$ (called front level set) is used to define the position of the crack tip (Fig. 4). Both level sets are discretized

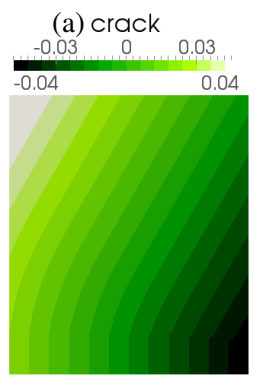

(b) front

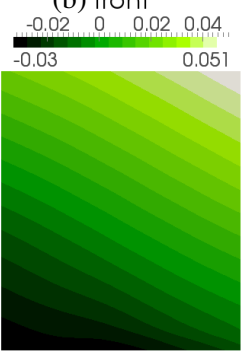

(c) iso-zeros

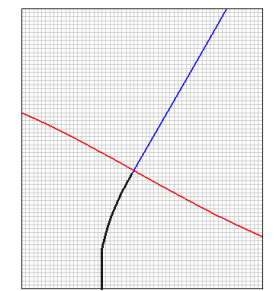

Fig. 4 Crack representation by level sets on a regular grid: (a) crack $\phi$, (b) front $\psi$ and (c) iso-zero of $\phi$ (blue and black) and $\psi$ (red) defining the crack geometry (black)

using finite differences on an auxilary regular grid following [34], rather than on the finite element meshes for the following reasons: (a) it is possible to use a localized grid around the crack since there is no need to define the level set over the entire domain. (b) one can use a structured grid on which existing optimized solvers (like the projection methods of [14] used herein) can be used to perform propagation, (c) since more than one mesh is used in the same area, one would face up to different level sets defined on different grids because of numerical errors.

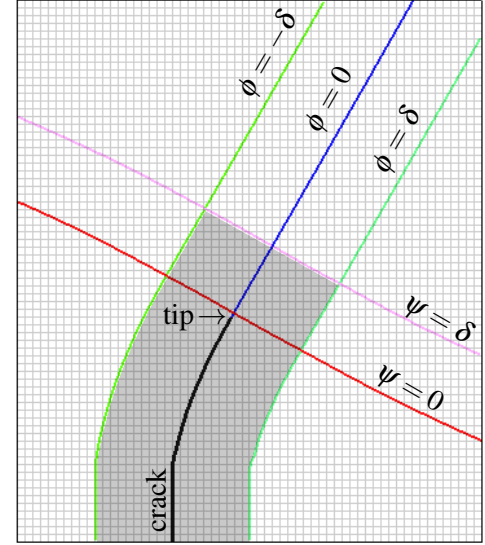

Fig. 5 Level sets based definition of the shape of local model (gray) The local model corresponds to values of $\phi$ ranging from $\phi=-\delta$ to $\phi=\delta$ (green lines) and values of $\psi$ lower than $\delta$ (pink line).

The local domain is defined such as few elements form a layer on each side of the crack. Again, level sets is a very convenient tool to play that role. A geometric parameter $\delta$ is defined as half the width of the local domain. This parameter depends on the element size near the crack. In practice it is choosen as twice the characteristic element size. Finally, the local domain is defined by the set of elements whose center $x_{c}$ satisfies $-\delta \leq \phi\left(x_{c}\right) \leq \delta$ and $\psi\left(x_{c}\right) \leq \delta$, as shown figure 5 . Let us notice that even when the enrichments and shape of the local model are updated, the operator and solver of the global model remain the same.

\section{A first static crack analysis: edge crack plate under uniaxial stress}

In this section we first consider a static mode I straight crack problem in order to study the convergence of the proposed non-intrusive method. We analyse an edge-cracked plate, as shown in Figure 6(a), subjected to a uniform tensile stress $\sigma=10 \mathrm{MPa}$. The plate has a length $L=17 \mathrm{~mm}$ and width $w=7 \mathrm{~mm}$. A rather small crack size $a=1 \mathrm{~mm}$ is considered. The problem has thus two different scales. The structure is considered to be in plane strain conditions, with a Poisson's ratio equal to $v=0.3$ and Young's modulus $E=200 \mathrm{GPa}$. The initial global model has a regular mesh made of $25 \times$ 59 bilinear quadrangular elements, with a Gauss quadrature following [29]. The shape of domain for the local model is parametrized using the geometric parameter $\delta=2 h$ ( $h$ being the element size). The global fem mesh and the local model are respectively plotted in Figures 6(b) and 6(c). For such a problem, the value of $K_{I}$ can be accurately approximated by the one that holds for an infinite plate, corrected by a factor 


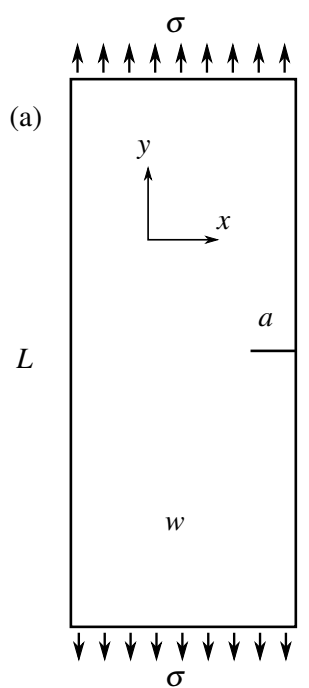

(b)

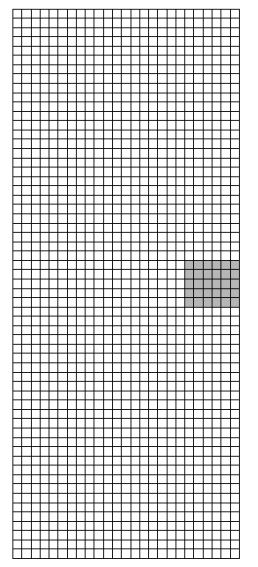

(c)

Fig. 6 Edge crack plate under uniaxial stress: (a) geometry and loading; (b) global FEM model (the local region in gray) and (c) local model including and X-FEM grid (crack in red) and the analytical patch (black)

depending on the ratio $\frac{a}{w}[8]$ :

$$
\begin{aligned}
K_{I}^{r e f}=\sigma & \sqrt{a \pi}\left[1.12-0.231 \frac{a}{w}+10.55 \frac{a^{2}}{w}\right. \\
& \left.-21.72 \frac{a}{w}^{3}+30.39 \frac{a^{4}}{w}\right]=0.70154 \mathrm{MPa} \sqrt{\mathrm{m}} \\
K_{I I}^{r e f}= & 0 \mathrm{MPa} \sqrt{\mathrm{m}}
\end{aligned}
$$

First the global FEM problem is solved without accounting for the crack, using the global solver alone. The corresponding solution is independent from the $x$-axis as shown in Figure 7(a). Next, the problem is solved using the proposed local/global non-intrusive coupling. The resulting Stress Intensity Factors (SIFs) are accurately estimated, with a relative error of $0.0689 \%$ on $K_{I}$ with respect to $K_{I}^{r e f}$ reference values. The associated global and local displacement fields are respectively plotted in figure 7(b) and 7(c). The solution resulting from this local/global strategy corresponds to the union of the local model and the global model excluding the local part of the global model, as show Figure 7(d). One can notice that even if the crack has mainly a local effect, it still causes a stress redistribution at the scale of the global model, which can not be taken into account with oneway zoom approaches [11]. With such a local/global coupling, the global solution is exact far from the crack. Furthermore, it is achieved without modifying the mesh nor the solver of the global model. Such methods may be of great interest for engineers, as it yields a quasi exact solution without resorting to a specialized solver throughout the model.

The convergence is now analysed thanks to two indicators. First a stagnation relative error on both models, which only consists in a measure of the distance between the solutions of two consecutive iterations. This basic stagnation
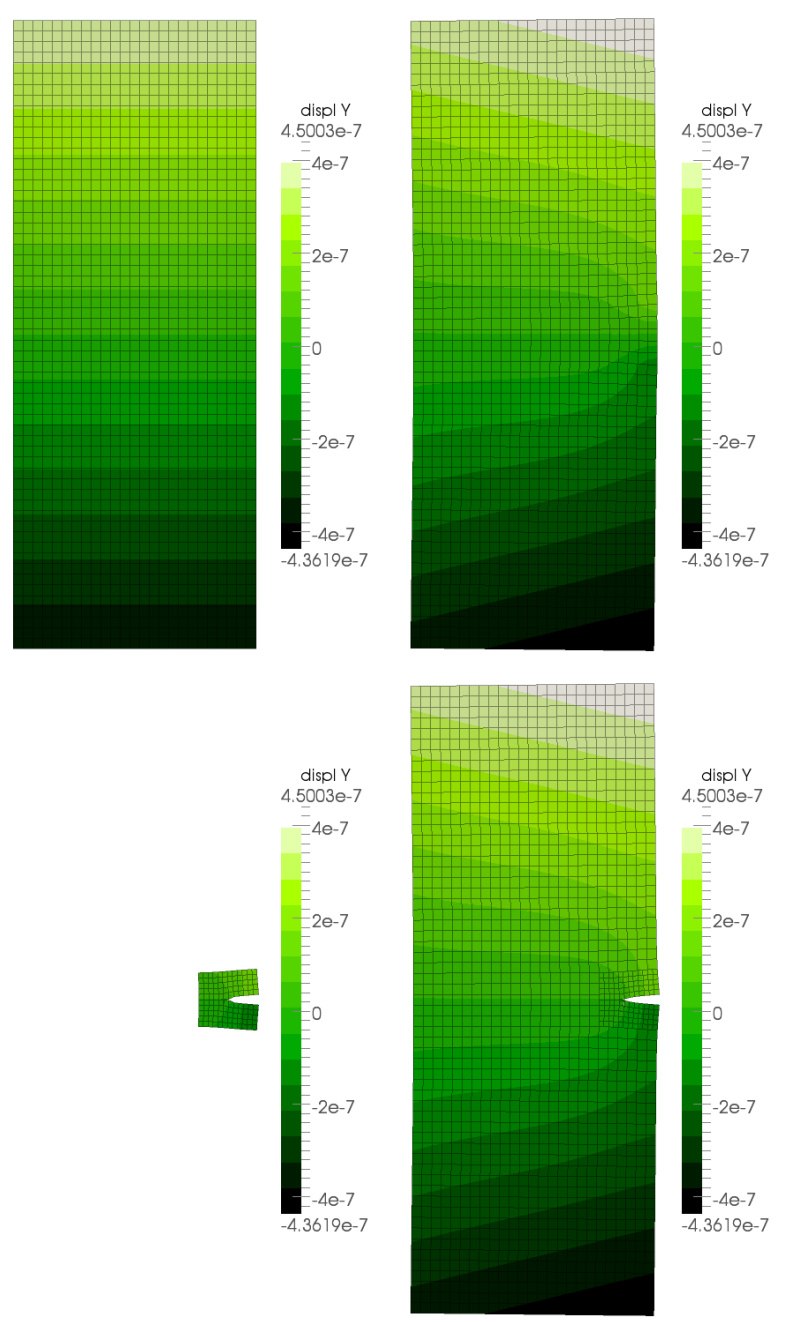

Fig. 7 Vertical displacement (in m) of: the global model without (a) and with (b) the coupling with the local model; the local model (c) and the local/global solution which corresponds to the substitution of the local model in the global one (d). (warp ampl. factor: $10^{3}$ )

indicator was shown to be a good error indicator in a previous study [29]. Next, as mode $I$ and mode $I I$ stress intensity factor reference values exist, an exact error measure can be defined as:

$e_{S I F}^{2}=\frac{\left\|\mathbf{s}-\mathbf{s}^{r e f}\right\|^{2}}{\left\|\mathbf{s}^{r e f}\right\|^{2}} \quad$ with $\quad \mathbf{s}=\left[\begin{array}{c}K_{I} \\ K_{I I}\end{array}\right]$ and $\quad \mathbf{s}^{r e f}=\left[\begin{array}{l}K_{I}^{r e f} \\ K_{I I}^{r e f}\end{array}\right]$

These indicators are plotted as a function of the iteration number in Figure 8. The exact error reaches an asymptotic value, which corresponds to the fact that the exact error includes the finite element approximation error, the error caused by the truncation in the William's expansion and by the size of the analytical patch (see [37,29] for a deeper analysis). One can notice that after 20 iterations, the solution has almost converged. Let us recall that an iteration of the multigrid solver consists in one full resolution on the global model with the standard solver and a few smoothing itera- 


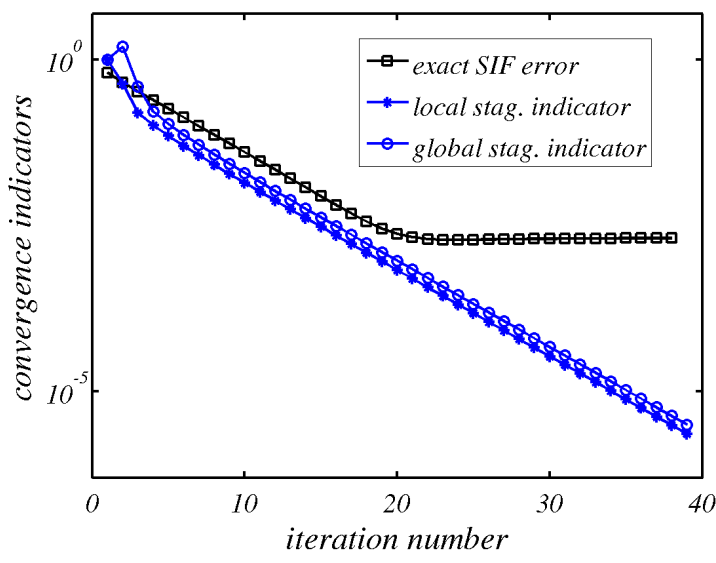

Fig. 8 Convergence of the non-intrusive local/global coupling.

tions (5 or 6) of an iterative solver (here Conjugate Gradient) on the local model. Obviously the number of iterations to convergence strongly depends on the problem, and more precisely on the separability of the scales of the global and local models. The more the scales are distinct, the less iterations it takes to converge. Finally, the stagnation indicators on both models (which is definitely not resource demanding) still remain reliable convergence indicators in the context of a multigrid solver. In the sequel, a stopping criteria based of these stagnation indicators is used.

\section{Curvilinear crack propagation simulation: Ingraffea and Grigoriu's experiment}

In this section, the ability of the proposed non-intrusive coupling technique to simulate the propagation of a curved mixed mode crack is studied. A non intrusive simulation of Ingraffea and Grigoriu tests [22] is performed for this purpose. A Polymethyl methacrylate (PMMA) plate with an initial notch is considered under three point bending test. The hypothesis of plane stress is assumed. The crack path is modified by the presence of open holes see Fig.9.

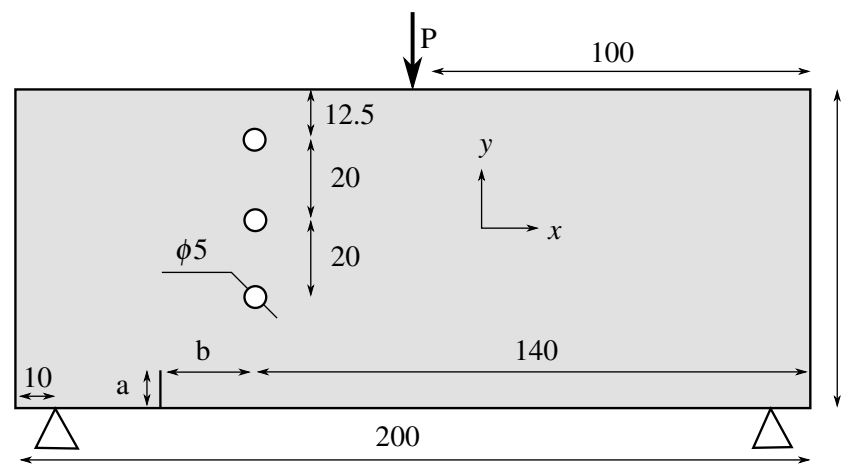

Fig. 9 Definition of the geometry, loading and initial notch
It additionally depends on the location and size of the initial notch. Therefore two different notch geometries are considered: case (1) $a=10, b=15$ and case (2) $a=20$, $b=10$. A brittle fracture criterion is used for propagation. The crack grows of an increment set to $d a=1$ per iteration. The direction is determined by the maximum hoop stress criterion, which reads:

$\theta_{C}=2 \tan ^{-1}\left[\frac{1}{4}\left(\frac{K_{I}}{K_{I I}}-\operatorname{sign}\left(K_{I I}\right) \sqrt{\left(\frac{K_{I}}{K_{I I}}\right)^{2}+8}\right)\right]$

For the simulation, an unstructured mesh of three-node, linear triangular elements is considered for the global FEM model. Figure 10 presents the crack path computed using the proposed non-intrusive method (in red) as compared to the experimental observations provided by [22] (in black). One

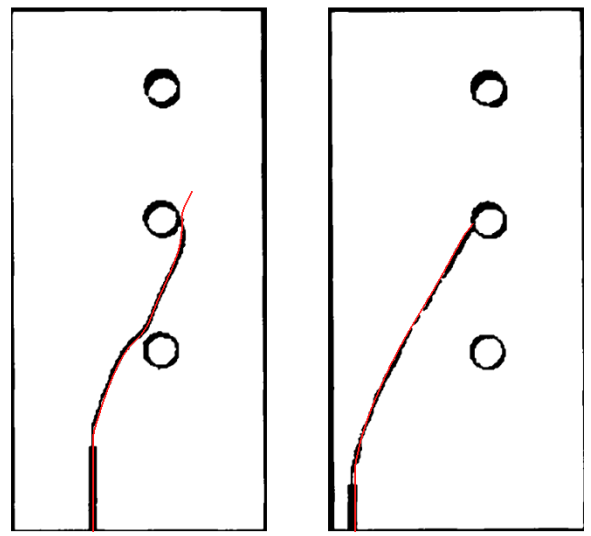

Fig. 10 Comparison of the simulated crack path with the experimental observations: case 1 (left) and case 2 (right)

can see that the crack path is very accurately predicted, even with a rather coarse unstructured initial mesh. One must mention that, in Case 1, the crack does not hit the second hole as it seems to be the case experimentally. In practice, the second hole is avoided by the crack in most simulations that the authors found in the wide literature studying this classical benchmark [39, 19,20,14].

Remark. Because of the holes, the crack path has a complex non rectilinear geometry, especially in case 1 . In the local model, an arbitrary geometry of the crack path is easily handled by X-FEM. However, the fields used in the analytical patch are those holding for a small straight segment located at the near-tip of the crack (see (8) and (9)) In the examples here, it is assumed that the crack path in the region of the patch is chosen to be the secant line joining the crack tip to the intersection of the crack path with the boundary of the patch. If the radius of curvature of the crack could not be neglected within the analytical patch, two possibilities exist that can take into account the curvature of the crack. 
The first one consists in computing and adding a correction to the Williams' expansion, so that it is correct for curved cracks, as described in [38]. Another way to better describe curvilinear crack, is to refine the mesh. Here, the method is based on multigrid solver. As it was shown in [29], one can consider the size of the analytical patch as small as necessary, without remeshing, and without reducing the accuracy of the SIF estimation. Indeed, one has just to build additional finer grids until the first hypothesis (straight crack) is lawfulacceptable inside the analytical patch. Such an adaptive refinement approach remains to be developed, and needs a deeper analysis. Finally, one can notice that the same limitation stands for standard X-FEM simulations, since tip enrichments functions and interaction integral SIF extraction are based on the same assumptions.

The solution at the $31^{\text {th }}$ propagation step is illustrated in Figure 11. The horizontal displacement of the global FEM

(a)

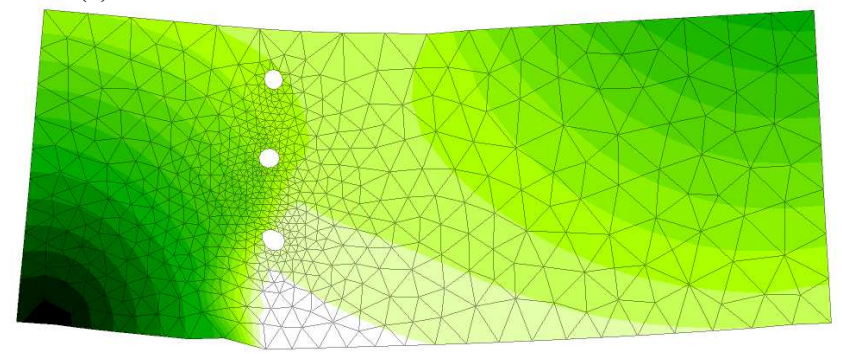

(c)
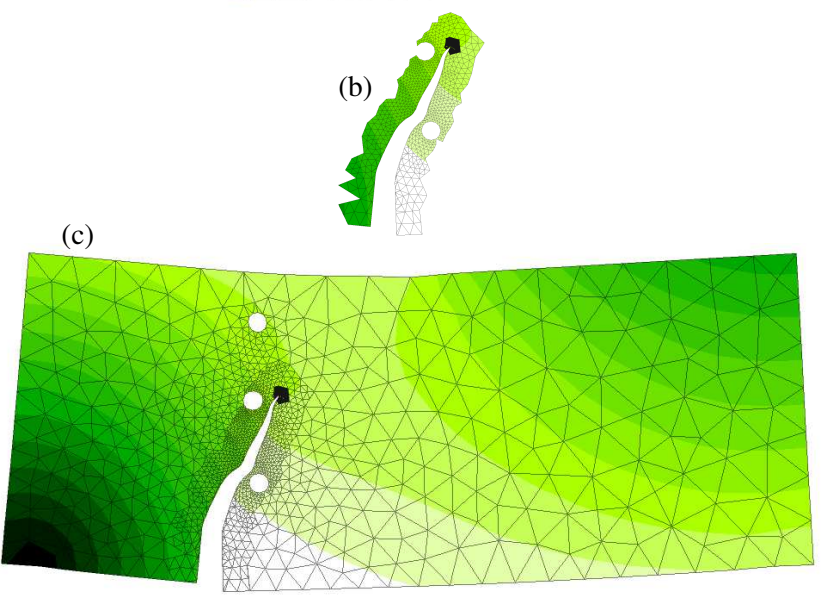

Fig. 11 Graphical representation of the converged solutions at the $31^{\text {th }}$ propagation step: (a) the solution of the global FEM model; (b) the local model based on DEK-FEM, with the analytical patch (in black) for direct estimation of SIFs and (c) the solution resulting from the substitution of solution of the local model in the initial mesh.

model is plotted in Figure 11(a). The local/global coupling makes the global model behave as if it was able to account for the crack. At least outside the local zone, in which the valid solution is that of the local model 11(c), as depicts Figure 11(c).
The shape definition based on levelsets and described in section 3.3 is used to automatically uptdate the geometry of the domain over which the local model applies during propagation. The characteristic element size of the global mesh being $h=4.7 \mathrm{~mm}$, the local model geometric parameter $\delta$ is thus set to $\delta=2 h=9.4 \mathrm{~mm}$. The shape and mesh of the local model and its analytical domain are plotted in Figure 12 for differents propagation steps (1, 7, 13, 19, 25 and 31). According to this technique, the question of the definition

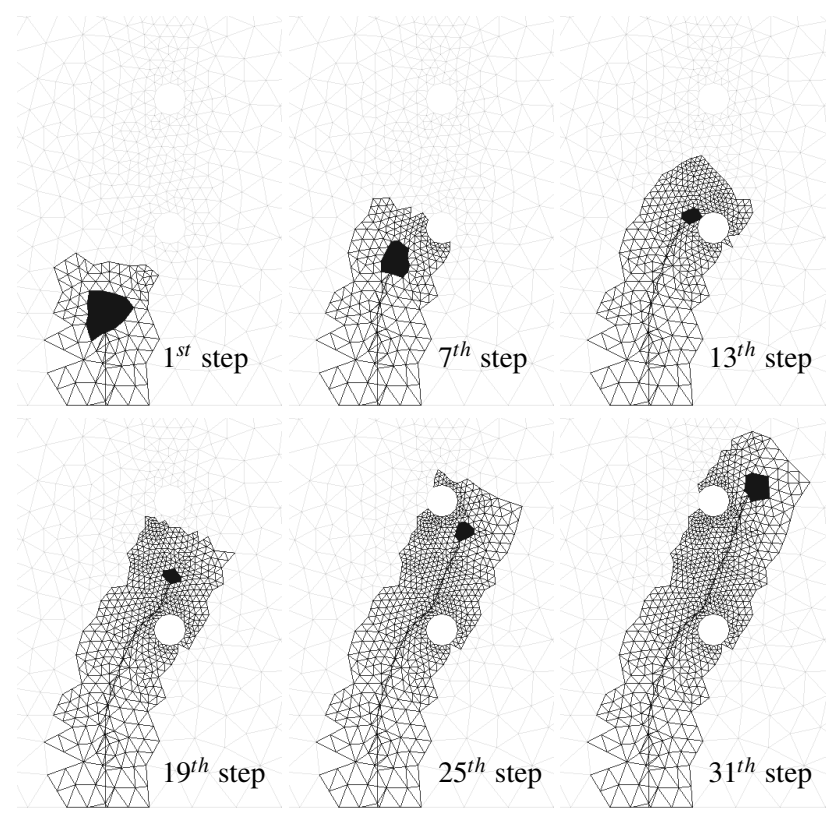

Fig. 12 Levelset-based adaptivity of the grid geometries during propagation: in black, the shape of the analytical patch for direct SIF extraction.

of geometries of global and local domains, which remains an open question for other types of local phenomena, is for fracture problem easily solved.

\section{Experimental/numerical study of an open hole plate in compression.}

In this section, we show that the non-intrusiveness condition is not only an advantage for the code development or code coupling. It is also an advantage that improves, for instance, the link between numerical simulations and experimental observations. An open hole PMMA plate under uniaxial compression is studied to illustrate this point, see Fig.13. This specimen was initially used for the analysis of dynamic mixed mode crack propagation in [18]

Experimental results for this plate have been obtained at LaMCoS (INSA de Lyon). The quasi-static propagation from a notch in the open hole is recorded by a digital camera. 


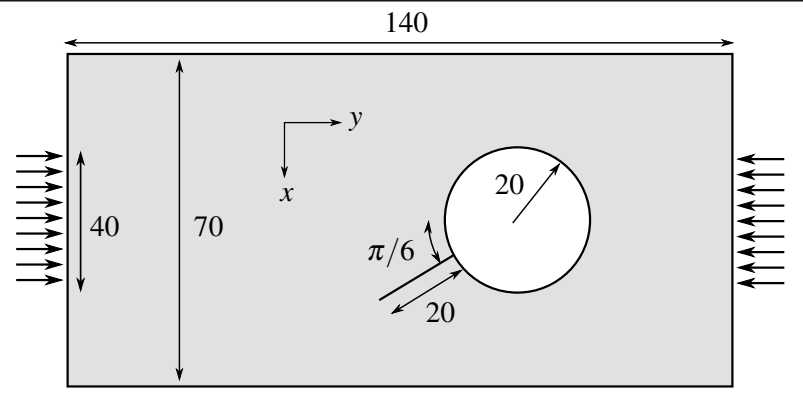

Fig. 13 Definition of the geometry and loading of the studied test case (in $\mathrm{mm}$ ).

The surface of the plate is covered by a Black/White paint pattern, in order to measure the experimental displacement field by digital image correlation (DIC). A finite elementbased DIC is performed as described in [5], with a global bilinear quadrilateral mesh presented in Figure 14. In this fig-

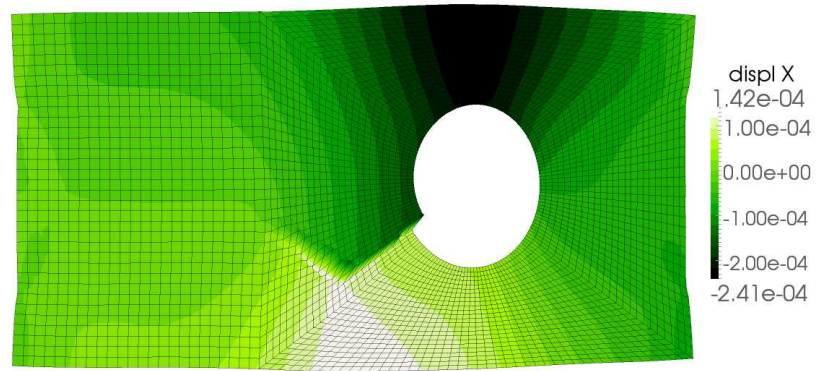

Fig. $14 x$-component of the experimental displacement field (in m) measured with a FE-DIC method.

ure the experimental displacement field is represented. Only a basic FE-DIC is performed, which explains the smoothed discontinuity of the displacement.

In a second step, the non intrusive coupling is used to simulate this problem, with the same mesh. A brittle fracture criterion is used again. A crack growth increment of $d a=1 \mathrm{~mm}$ and the aforementioned maximum hoop stress criterion are considered. Using the same mesh for DIC and simulation has the advantage that one can easily prescribe the experimental boundary conditions for the simulation, expressed here directly using the measured experimental displacement along the boundaries. This is further performed directly without interpolation and without modifying the degrees of freedom connectivity.

Figure 15 presents the local/global solution (including the global, the local and the full solution) after 15 propagation steps. Qualitatively, one can notice that the simulated displacement on the global model is very similar to the measured one. One can also compare the crack paths, which are well predicted by the local/global simulation with respect to the experimental images, see Fig. 16.
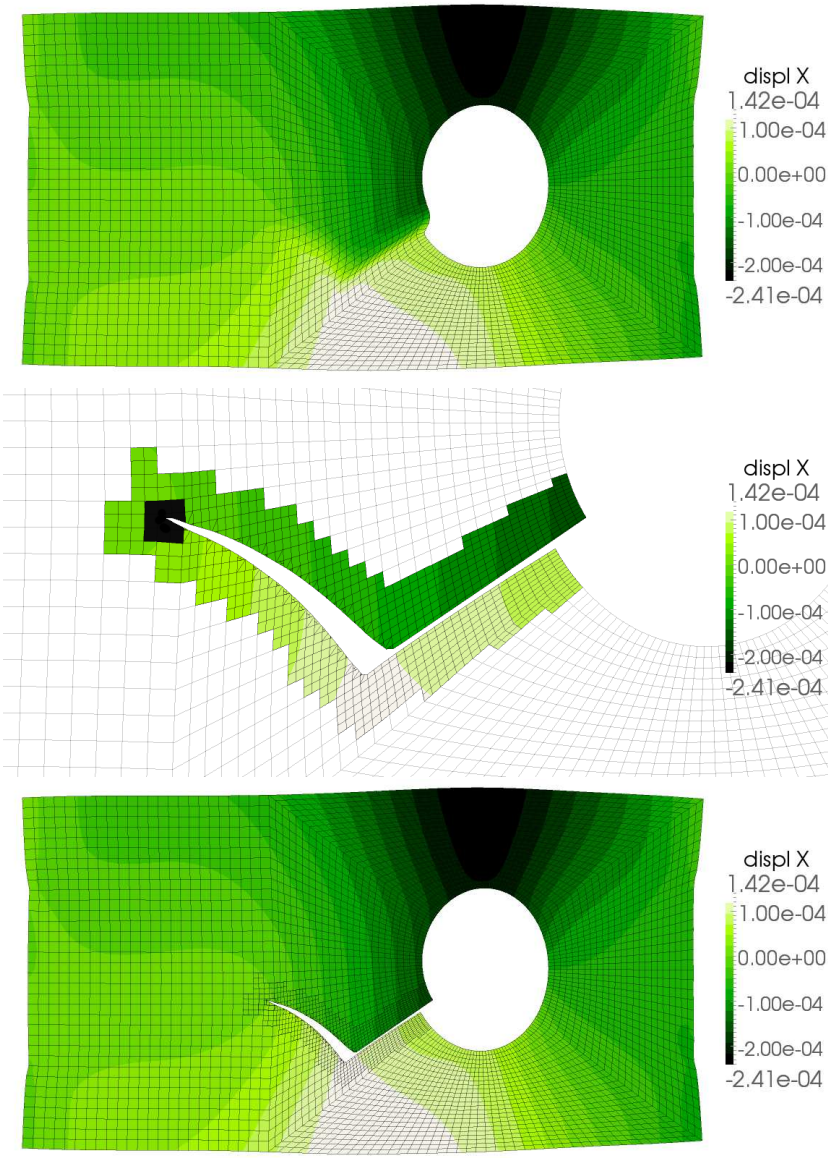

Fig. 15 Graphical representation of the solution algorithm: (a) nonintrusive solves on the initial mesh; (b) the local model based on DEKFEM and (c) the solution resulting from the substitution of solution of the local model in the initial mesh.

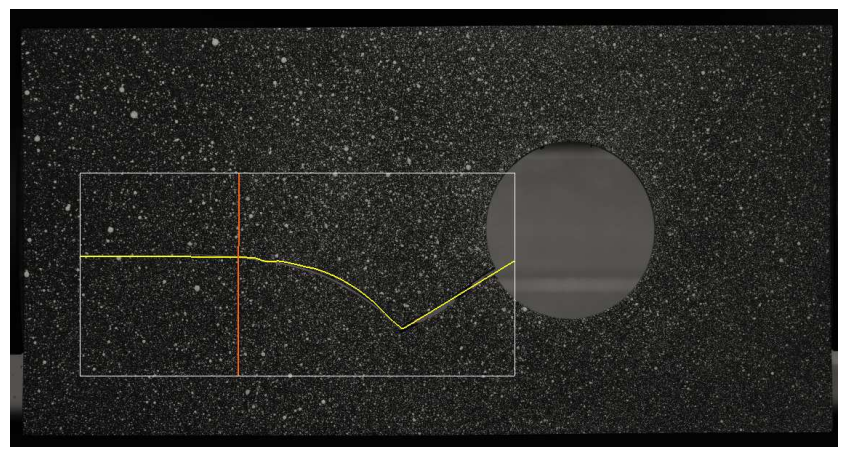

Fig. 16 Superposition of the crack path on the deformed digital image.

But here, one can go a step further, since the meshes are identical, with the same connectivity. It is therefore possible to directly compare simulated and measured displacement maps to quantify the accuracy of the simulation with respect to experiments, either by a global error measure $e\left(\mathbf{U}_{g}, \mathbf{U}_{\text {meas }}\right)$ in the $L^{2}$ norm, and also by a relative discrepancy map $\mathbf{d}\left(\mathbf{U}_{g}, \mathbf{U}_{\text {meas }}\right)$ 
$e\left(\mathbf{U}_{g}, \mathbf{U}_{\text {meas }}\right)=\frac{\left\|\mathbf{U}_{G}-\mathbf{U}_{\text {meas }}\right\|}{\left\|U_{\text {meas }}\right\|}$

$\mathbf{d}\left(\mathbf{U}_{g}, \mathbf{U}_{\text {meas }}\right)=\frac{\left|\mathbf{U}_{G}-\mathbf{U}_{\text {meas }}\right|}{\max \left(\left|U_{\text {meas }}\right|\right)}$

These indicators are only computed over the local region of the global model. Indeed, after convergence, we know that the global solution under the local model is not physical. It is therefore logical that the high levels of error in the local region (top). If the elements that are in the local region, are not considered (bottom), the relative error quantifies the distance between simulated and experimental displacement fields. For the same step, the global error measure $e$ is equal
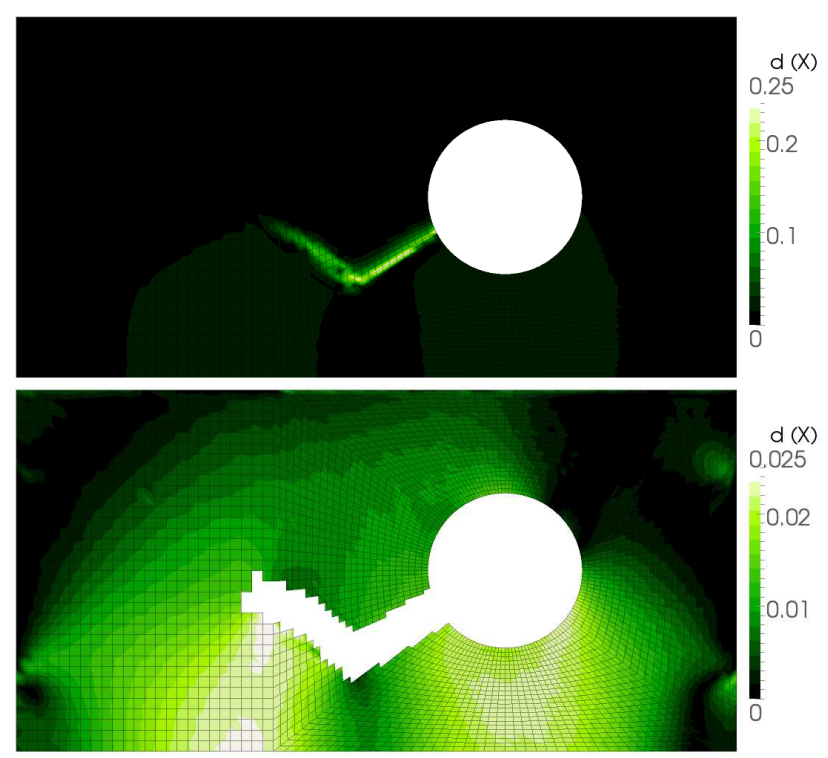

Fig. 17 Direct numerical/experimental displacement field comparison: relative discrepancy map $\mathbf{d}$ on the $x$-component of the displacement with (top) and without (bottom) considering the local region.

to 0.0342 . One can easily imagine extensions of such a work to identification.

Non-intrusive techniques not only simplify coding concerns, but it also provides a lot of flexibility for the user. As an example, we showed it may facilitate the dialogue between experimental and numerical analysis.

\section{Conclusion}

In this paper a local/global non-intrusive coupling method was proposed for the analysis a local cracking and its propagation using a global linear elastic model not intended for that purpose. The proposed algorithm is based on a multigrid and extended finite element method proposed recently in [35,29]. It is said non-intrusive since neither the connectivity, nor the solver of the global model are modifyed during propagation. From the viewpoint of the global model, the contribution of the local model (and thus the crack) only consists in additional nodal efforts which make it compatible with a large number of softwares.

Although the method can be applied to a standard XFEM method, it is here applied to a variant which has the advantage to yield directly the stress intensity factors and higher order terms [29]. Therefore, the model involves at least three nested models: the global model, the local model and the analytical patch based on a truncated William's expansion. It was shown, in this paper, that the multigrid $W$ cycle proposed in [29] to combine these 3 models, can be replaced by a two-grid $V$-cycle which makes one parameter less. An automatic adaptive shape of the local domain is proposed, based on the level set based crack description. The non-intrusivness is preserved even when the crack propagates and when the shape of doamin of the local model evolves

As a short term perspective, it could be interesting to used such a method to truly couple a real commercial FEA software and a reasearch piece of code, as it was done in [12]. As mentionned before, another extension of this work would be to develop an adaptativity of the grid hierarchy, following [6].

The extension of this word to 3D crack growth with a standard X-FEM does not presents theoretical difficulties, as $\mathrm{X}-\mathrm{FEM}$ multigrid as already be extended to 3D [35]. Concerning the sif extraction, it is less straightforward. Indeed, the definition of the williams' modes are more complicated. They need to be defined in each plane orthogonal to the crack front, and based on a 1D finite element interpolation (mesh + shape functions) of the generalized SIF along the crack front, which may require further developments.

Finally, since the global displacement does not model the evolving discontinuity, the proposed algorithm could also be used to locally enhance a global reduced order model [23]. 
Acknowledgements Jean-Charles Passieux would like to acknowledge the financial support of the Agence Nationale de la Recherche under grant ICARE ANR-12-MONU-0002. The support of the Agence Nationale de la Recherche under grant RUPXCUBE ANR-09-BLAN0009-01 RUPX CUBE is also gratefully acknowledged by the authors.

\section{References}

1. Allix, O., Gendre, L., Gosselet, P., Guguin, G.: Non-intrusive coupling: An attempt to merge industrial and research software capabilities. In: Recent developments and innovative application in computational mechanics, chap. 15, pp. 125-133. Springer (2011)

2. Barsoum, R.: Application of quadratic isoparametric elements in linear fracture mechanics. Int. J. Fracture 10, 603-605 (1974)

3. Ben Dhia, H., Jamond, O.: On the use of xfem within the arlequin framework for the simulation of crack propagation. Computer methods in applied mechanics and engineering 199(21-22), 1403$1414(2010)$

4. Berger-Vergiat, L., Waisman, H., Hiriyur, B., Tuminaro, R., Keyes, D.: Inexact schwarz-algebraic multigrid preconditioners for crack problems modeled by extended finite element methods. International Journal for Numerical Methods in Engineering 90(3), 311-328 (2012)

5. Besnard, G., Hild, F., Roux, S.: finite-element displacement fields analysis from digital images: Application to portevinle chtelier bands. Experimental Mechanics 46(6), 789-803 (2006)

6. Biotteau, E., Gravouil, A., Lubrecht, A., Combescure, A.: Three dimensional automatic refinement method for transient small strain elastoplastic finite element computations. Computational Mechanics 49(1), 123-136 (2012)

7. Brandt, A.: Multigrid Techniques: 1984 Guide with applications to fluid dynamics. The Weizmann Institute of Science: Rehovot, Israel (1984)

8. Bui, H.: Mécanique de la Rupture Fragile. Masson, Paris, France (1978)

9. Chahine, E., Laborde, P., Renard, Y.: A non-conformal extended finite element approach: Integral matching xfem. Applied Numerical Mathematics 61(3), 322-343 (2011)

10. Chiaruttini, V., Feyel, F., Chaboche, J.L.: A robust meshing algorithm for complex $3 \mathrm{~d}$ crack growth simulations. In: European Congress on Computational Mechanics, Venice, Italy. (2010)

11. Cormier, N., Smallwood, B., Sinclair, G., Meda, G.: Aggressive submodelling of stress concentrations. International Journal for Numerical Methods in Engineering 46(6), 889-909 (1999)

12. Gendre, L., Allix, O., Gosselet, P.: Non-intrusive and exact global/local techniques for structural problems with local plasticity. Computational Mechanics 44, 233-245 (2009)

13. Gendre, L., Allix O. Gosselet, P.: A two-scale approximation of the schur complement and its use for non-intrusive coupling. International Journal for Numerical Methods in Engineering 87, 889905 (2011)

14. Geniaut, S., Galenne, E.: A simple method for crack growth in mixed mode with $\mathrm{x}$-fem. International Journal of Solids and Structures 49(15-16), 2094-2106 (2012)

15. Gerstenberger, A., Tuminaro, R.: An algebraic multigrid approach to solve XFEM based fracture problems. International Journal for Numerical Methods in Engineering p. online first (2012)

16. Glowinski, R., He, J., Lozinski, A., Rappaz, J., Wagner, J.: Finite element approximation of multi-scale elliptic problems using patches of elements. Numer. Math. 101(4), 663-687 (2005)

17. Gravouil, A., Moës, N., Belytschko, T.: Non-planar crack growth by the extended finite element and level sets. Part II: Level-Set update. International Journal for Numerical Methods in Engineering 53(11), 2569-2586 (2001)
18. Grégoire, D., Maigre, H., Réthoré, J., Combescure, A.: Dynamic crack propagation under mixed-mode loading - comparison between experiments and $x$-fem simulations. International Journal of Solids and Structures 44(20), 6517-6534 (2007)

19. Guidault, P.A., Allix, O., Champaney, L., Cornuault, C.: A multiscale extended finite element method for crack propagation. Computer Methods in Applied Mechanics and Engineering 197(5), 381-399 (2008)

20. Häusler, S.M., Lindhorst, K., Horst, P.: Combination of the material force concept and the extended finite element method for mixed mode crack growth simulations. International Journal for Numerical Methods in Engineering 85(12), 1522-1542 (2011)

21. Holl, M., Loehnert, S., Wriggers, P.: An adaptive multiscale method for crack propagation and crack coalescence. International Journal for Numerical Methods in Engineering 93(1), 23-51 (2013)

22. Ingraffea, A., Grigoriu, M.: Probabilistic fracture mechanics: A validation of predictive capability. Tech. Rep. 90-8, Department of Structural Engineering, Cornell University (1990)

23. Kerfriden, P., Passieux, J., Bordas, S.: Local/global model order reduction strategy for the simulation of quasi-brittle fracture. International Journal for Numerical Methods in Engineering 89(2), 154-179 (2012)

24. Kim, D.J., Pereira, J., Duarte, C.: Analysis of three-dimentional fracture mechanics problems: A two-scale approach using coarsegeneralized fem meshes. International Journal for Numerical Methods in Engineering 81, 335-365 (2010)

25. Lozinski, A.: Méthodes numériques et modélisation pour certains problèmes multi-échelles. Habilitation à diriger des recherches, Université Paul Sabatier (2010)

26. Lozinski, A., Pironneau, O.: Numerical zoom for localized multiscales. Numerical Methods for Partial Differential Equations 27, 197-207 (2011)

27. Moës, N., Dolbow, J., Belytschko, T.: A finite element method for crack growth without remeshing. International Journal for Numerical Methods in Engineering 46(1), 131-150 (1999)

28. Parsons, I., Hall, J.: The multigrid method in solid mechanics: part I-algorithm description and behaviour. International Journal for Numerical Methods in Engineering 29, 719-737 (1990)

29. Passieux, J.C., Gravouil, A., Réthoré, J., Baietto, M.C.: Direct estimation of generalized stress intensity factors using a three-scale concurrent multigrid $x$-fem. Int. J. Numer. Meth. Eng. 85(13), 1648-1666 (2011)

30. Passieux, J.C., Ladevèze, P., Néron, D.: A scalable time-space multiscale domain decomposition method: adaptive time scale separation. Comput. Mech. 46(4), 621-633 (2010)

31. Pereira, J., Duarte, C.: Extraction of stress intensity factors from generalized finite element solutions. Engineering Analysis with Boundary Elements 29, 397-413 (2005)

32. Pereira, J., Kim, D., Duarte, C.: A two-scale approach for the analysis of propagating three-dimensional fractures. Computational Mechanics 49(1), 99-121 (2012)

33. Pierrès, E., Baietto, M.C., Gravouil, A.: A two-scale extended finite element method for modelling $3 \mathrm{~d}$ crack growth with interfacial contact. Computer Methods in Applied Mechanics and Engineering 199(17-20), 1165-1177 (2010)

34. Prabel, B., Combescure, A., Gravouil, A., Marie, S.: Level set Xfem non-matching meshes: Application to dynamic crack propagation in elastic-plastic media. International Journal for Numerical Methods in Engineering 69, 1553-1569 (2006)

35. Rannou, J., Gravouil, A., Baietto-Dubourg, M.C.: A local multigrid X-FEM strategy for 3-D crack propagation. International Journal for Numerical Methods in Engineering 77, 581-600 (2008)

36. Rannou, J., Gravouil, A., Combescure, A.: A multi-grid extended finite element method for elastic crack growth simulation. European Journal on Computational Mechanics 16, 161-182 (2007) 
37. Réthoré, J., Roux, S., Hild, F.: Hybrid analytical and extended finite element method (HAX-FEM): A new enrichment procedure for cracked solids. International Journal for Numerical Methods in Engineering 81(3), 269-285 (2010)

38. Réthoré, J., Roux, S., Hild, F.: Mixed-mode crack propagation using a hybrid analytical and extended finite element method. Comptes Rendus de Mécanique 338, 201-126 (2010)

39. T.N. Bittencourt P.A. Wawrzynek, A.I., Sousa, J.: Quasi-automatic simulation of crack propagation for $2 \mathrm{~d}$ lefm problems. Engineering Fracture Mechanics 55(2), 321-334 (1996)

40. Williams, M.: On the stress distribution at the base of a stationary crack. ASME Journal of Applied Mechanics 24, 109-114 (1957) 